Slavica

bruxellensia

\section{Slavica bruxellensia}

Revue polyphonique de littérature, culture et histoire

slaves

$7 \mid 2011$

Les Slaves, le Midi et l'Orient

\title{
Entretien avec Jean-Charles Ducène
}

Dorota Walczak et Katia Vandenborre

\section{OpenEdition}

Journals

Édition électronique

URL : http://journals.openedition.org/slavica/662

DOI : 10.4000/slavica.662

ISSN : 2034-6395

Éditeur

Université libre de Bruxelles - ULB

\section{Référence électronique}

Dorota Walczak et Katia Vandenborre, «Entretien avec Jean-Charles Ducène », Slavica bruxellensia [En ligne], 7 | 2011, mis en ligne le 15 avril 2011, consulté le 21 avril 2019. URL : http:// journals.openedition.org/slavica/662 ; DOI : 10.4000/slavica.662

Ce document a été généré automatiquement le 21 avril 2019.

\section{(c) (i) (9)}

Les contenus de Slavica bruxellensia sont mis à disposition selon les termes de la Licence Creative Commons Attribution - Pas d'Utilisation Commerciale - Pas de Modification 3.0 France. 


\title{
Entretien avec Jean-Charles Ducène
}

\author{
Dorota Walczak et Katia Vandenborre
}

\section{Présentation}

1 Jean-Charles Ducène est licencié en Philologie et histoire orientales (ULB, 1994) et docteur en Philosophie et lettres de cette même université (2002). Il a à cette occasion soutenu une thèse ayant pour objet l'édition, la traduction et le commentaire du Kitāb dalā'il alqibla d'Ibn al-Qâss (IV ${ }^{\mathrm{e}} / \mathrm{X}^{\mathrm{e}}$ siècle). Depuis septembre 2004, il enseigne à l'ULB l'histoire des peuples arabes et musulmans. En décembre 2010, il a obtenu son Habilitation à diriger des recherches, l'à EPHE, à Paris.

Ses recherches portent notamment sur la géographie et la cartographie arabes médiévales abordées sous deux axes : d'abord comme témoins de l'évolution des idées et de la représentation du monde, mais aussi comme sources d'informations positives en géographie historique. Il s'intéresse aussi à la manière dont les érudits européens ont utilisé les auteurs orientaux de la Renaissance au XVIII ${ }^{\mathrm{e}}$ siècle.

\section{Entretien}

Dorota Walczak: Nous tous, Européens, devons une grande part de notre savoir aux sciences arabes de l'Antiquité et du Moyen Âge. Cela est incontestable. Je voudrais commencer par une question un peu naïve et un peu provocatrice: Qu'est-ce que la géographie et la cartographie arabe médiévales peuvent nous apprendre aujourd'hui ?

Jean-Charles Ducène : L'étude de cette littérature doit d'abord se comprendre comme l'étude d'une littérature ancienne et les acquis engrangés sont de l'ordre des connaissances historiques. Plus précisément, elle permet d'entrer dans deux domaines. D'abord, la géographie est premièrement la représentation du monde pour celui qui le conçoit, de sorte qu'en étudier l'évolution revient à faire de l'histoire des mentalités, mais aussi de l'histoire des sciences car les auteurs anciens avaient aussi le souci d'objectiver leur résultat pour rendre leur image en relation analogique avec le réel. Ensuite, les textes qui sont à notre disposition vont du VIII ${ }^{\mathrm{e}}$ jusqu'au $\mathrm{XV}^{\mathrm{e}}$ siècle, c'est 
dire que l'aspect diachronique est important et qu'en plus de pouvoir cerner l'évolution d'une image du monde, l'étude de la géographie arabe médiévale permet également de faire de la géographie historique sur le long terme. Par rapport aux littératures géographiques grecque, latine et chinoise, la spécificité de l'arabe est sa longévité et son ouverture sur les régions limitrophes du monde musulman. La littérature latine médiévale survit principalement sur l'héritage de l'Antiquité tardive. Elle devient originale surtout à partir du XII ${ }^{e}$ siècle, mais seulement pour l'Europe. La littérature géographique byzantine, hormis à cause des relations politiques, n'a pas développé une curiosité pour le monde qui lui est étranger. Une réflexion identique peut se faire pour la littérature chinoise qui, à part quelques relations de voyage - très intéressantes ceci dit - s'est centrée sur la Chine elle-même.

Les spécificités de la géographie arabe médiévale sont d'abord sa richesse et ensuite son domaine qui est quasi universel. Un savant russe de la première moitié du XIX siècle, Ignatij Juljanovič Kračkovskij, a dénombré plus de deux cent trente auteurs dont les œuvres peuvent être considérées comme abordant un aspect de la géographie. Et quant à son aire d'extension, elle va de la Scandinavie à la Corée, en passant par les populations finnoises de la Baltique et l'Afrique sub-saharienne! Voici deux exemples sur lesquels je travaille actuellement qui illustrent bien cette valeur. Je prépare l'édition d'un texte inédit d'al-Idrisi, un auteur du XII siècle qui décrit de manière précise les itinéraires routiers de la France et des régions limitrophes. Nous avons ainsi les localités principales qui étaient traversées par le voyageur sur un territoire allant de la Frise jusqu'à la Catalogne et la Lombardie. Dans les textes latins de cette époque, nous avons en tout et pour tout une dizaine d'itinéraires qui ont été conservés. En parallèle, je travaille sur un texte arabe $\mathrm{du} \mathrm{X}^{\mathrm{e}}$ siècle qui livre des descriptions topographiques détaillées de comptoirs commerciaux de la péninsule malaise et aussi la plus ancienne occurrence du gentilice «Batak » - les chasseurs de têtes - de Sumatra.

Finalement, pour répondre brièvement à votre question, je dirais que l'étude de ces textes permet, d'une part, d'entrer dans une représentation du monde et, d'autre part, de mettre à jour des informations géographiques anciennes, parfois si précises que l'archéologie peut s'en servir.

Katia Vandenborre: Attardons-nous encore sur la géographie puisque c'est elle qui a partiellement conditionné les contacts entre les Slaves et l'Orient. Dans quelle mesure la cartographie arabe médiévale peut-elle constituer un outil pour comprendre les contacts entre les Slaves et l'Orient?

JCD : On peut y voir un outil si l'on accepte l'idée que la carte est la résultante d'une accumulation d'informations et de la volonté de représenter graphiquement l'image du monde ou d'une de ses parties ainsi obtenue. Les problèmes mathématiques liés à la projection ne constituent ici qu'un aspect technique, secondaire. Ce que le cartographe a porté sur la carte ainsi que la position des objets représentent le résultat de ses connaissances, puis une part d'iconographie. Ceci dit, dans le cas précis qui nous occupe, nous n'avons que la cartographie d'al-Idrisi pour les pays slaves. Pour nous limiter à ces régions, on retrouve tout de même situées sur sa carte la Serbie, la Slavonie, la Croatie, la Bohème, la Pologne avec notamment les villes de Cracovie et de Gniezno ainsi que la Poméranie. Plus à l'est, se dessine une Russie, mais l'on constate que l'auteur a eu une information imprécise et lacunaire par endroits car ce sont essentiellement les territoires le long du Dniepr et du Dniestr, en Ukraine qui sont les mieux détaillés. D’ailleurs, la prépondérance des localités bordant ces fleuves et leur 
description dans le texte qui accompagne la carte laissent supposer une information passée par des commerçants exerçant dans la mer Noire. La Russie proprement dite est située entre un fleuve identifiable au Don et la Volga. C'est l'étude des strates d'informations, dont la représentation graphique constitue la carte, qui permet d'approcher les acteurs des contacts entre Slaves et Siciliens, dans ce cas précis. La forme du toponyme permet ainsi de cerner par quelle «bouche» il est passé. Par exemple, Tadeusz Lewicki a montré que la forme reprise par al-Idrisi pour le Danube ne provient pas d'une langue slave mais plutôt de la forme hongroise ou turque.

DW : Comment pourriez-vous décrire la distance et la proximité entre l'Orient et les pays slaves au Moyen Âge?

JCD : Il faut garder à l'esprit que les représentations mentales que se sont faites les orientaux, dans notre cas les Arabes et les Iraniens, se sont mises en place sur la longue durée, c'est-à-dire du $\mathrm{IX}^{\mathrm{e}} \mathrm{au} \mathrm{XV}$ siècle et ont forcément évolué. Les éléments qui ont joué dans le sens d'un rapprochement ont été d'abord des motifs commerciaux, et ceci dès l'origine. Par l'Asie centrale, les commerçants musulmans ont repris à leur compte les axes commerciaux mis en place par les commerçants sogdiens alors que, par le Caucase, ils avaient accès aux plaines pontiques. Les populations de l'Eurasie, Slaves, Hongrois, Bulgares, Russes, pouvaient leur fournir des fourrures dont les classes aisées à Bagdad étaient friandes - aussi étrange que cela puisse paraître quand on connaît le climat de la Mésopotamie - mais également des esclaves, en échange de pièces d'or. Remarquons que c'est justement la découverte de "trésors" constitués par ces monnaies, depuis l'Ukraine jusqu'en Scandinavie, qui ont permis de dresser la carte de ces routes commerciales. Ces relations drainent des informations qui sont recueillies justement par les géographes arabes "encyclopédiques» qui, au même moment, éprouvent une curiosité certaine pour ces peuples méconnus. Le mouvement de populations que connaît l'Eurasie au $\mathrm{X}^{\mathrm{e}}$ siècle, principalement d'est en ouest, $\mathrm{y}$ amène aussi des populations plus ou moins touchées par l'islam, comme les Petchenègues, de sorte qu'avec cette diffusion de leur religion, les Arabes et les Iraniens sont plus enclins à s'aventurer le long de la Volga. L'exemple le plus célèbre reste Ibn Fadlan qui se retrouve chez les Bulgares de la Volga dans la première moitié du $\mathrm{X}^{\mathrm{e}}$ siècle et nous livre la première description des rites funéraires païens slaves. Au XII siècle, on voit un voyageur andalou, Abû Hâmid, rester une vingtaine d'années entre la Volga et la Hongrie d'aujourd'hui, de sorte que les témoignages de ces auteurs, entre autres, alimentent la littérature arabe sur ces régions. Ce qui joue aussi c'est la diffusion de l'islam chez les Bulgares de la Volga et chez une partie des Hongrois. Mais il faut reconnaître que cette connaissance relative est aussi motivée par l'altérité: on s'intéresse à ces populations, à leurs mœurs, parce qu'elles sont très différentes pour l'auteur. Et comme le facteur religieux intervient, la christianisation des Hongrois, des Bulgares et des Slaves joue en sens inverse. Les invasions mongoles ne viennent, en réalité, que perturber momentanément la situation car dès la conversion à l'islam du khan de la Horde d'or et l'établissement d'un semblant d'État, les auteurs arabes nous fournissent à nouveau des informations sur ces populations, mais de manière bien plus parcimonieuse toutefois qu'auparavant. Ceci dit, j'ai découvert il y a peu la plus ancienne mention des coordonnées géographiques de la ville de Kiev, dans une table géographique yéménite de la fin du XIV ${ }^{\mathrm{e}}$ siècle, ce qui indique encore un intérêt pour ces régions. 
KV: Quels sont les premiers témoignages sur les rapports qu'entretenaient les Arabes avec les Slaves?

JCD : Les plus anciennes mentions de rapport se retrouvent dans les sources byzantines qui font état, au milieu du VII ${ }^{\mathrm{e}}$ siècle, de recrutements par les Arabes d'une population slave établie en Asie mineure et son établissement en Syrie. Au tout début du VIII siècle ce genre d'allusions apparaît aussi dans la littérature arabe. Il faut cependant attendre le deuxième quart $d u$ VIII $^{e}$ siècle pour que des expéditions militaires s'enfoncent au-delà du Caucase pour avoir de nouvelles mentions mais elles restent sporadiques et floues. On sait par un chroniqueur qu'à cette époque une population slave a été faite prisonnière et a été installée en Géorgie par les musulmans.

Par ailleurs, durant une période d'approximativement deux siècles, ce seront aussi les Slaves faits prisonniers et mis en esclavage par les Francs qui viendront alimenter les besoins en esclave de l'Espagne andalouse. Dans ce cas, c'est au détour d'une chronique que l'on croise un personnage au surnom « al-saqalabi » et que l'on se rend compte que seule l'onomastique a fini par garder trace de son origine. Un collègue algérien, Mohammed Méouak, a consacré une étude détaillée à ces Slaves, eunuques ou esclaves, employés par les Omeyyades d'Espagne, dont les fonctions allaient du domestique au responsable de haute charge. Pour en augmenter la valeur, ces malheureux étaient castrés avant d'être proposés à la vente. Quant à leur origine, il se peut que dans certains cas le terme de "saqalabi " ait plutôt eu pour sens "originaire d'Europe centrale », voire esclave blanc opposé à l'esclave noir, désigné par un autre terme.

Dans une moindre mesure, le hasard de l'existence a amené également des Slaves parmi les esclaves du califat de Baghdad. À partir du $\mathrm{X}^{\mathrm{e}}$ siècle, les besoins en troupes des armées musulmanes leur font recruter des soldats ou acheter des esclaves de diverses origines et c'est ainsi que nous retrouvons aussi des Slaves jusque dans les armées du calife fatimide d'Ifrîqiya, de Tunisie si vous voulez. On sait qu'il y avait à Palerme un quartier qui leur était réservé. L'origine de ces Slaves était l'Europe centrale et leur trafic débouchait en Méditerranée par la côte dalmate ou croate, alors que leur commerce était assuré notamment par des juifs et des Vénitiens. Si le destin de ces hommes peut maintenant nous effrayer, il faut aussi savoir qu'un esclave pouvait gravir les échelons du pouvoir tout en gardant sa condition servile, de même que les eunuques jouissaient d'une grande confiance de la part de leur maître. C'est ainsi que plusieurs grands commandants militaires des fatimides d'Ifrîqiya étaient slaves, comme par exemple le conquérant de l'Égypte et fondateur du Caire, Jawhar.

2 La majeure partie des témoignages sont toutefois des textes qui décrivent plus ou moins en détails les populations habitant l'est de l'Europe.

Ces textes ont une grande importance car ils éclairent une période mal connue par les textes latins, byzantins ou les premières chroniques slaves. Par exemple, à propos de ceux qui allaient devenir les Russes, les géographes arabes nous ont conservé des descriptions de cette population au moment où elle allait connaître sa slavisation. Ceci explique pourquoi depuis le XIX ${ }^{\mathrm{e}}$ siècle un certain nombre de savants slaves se sont tant intéressés à cette littérature géographique. Pratiquement, on sait qu'un livre dont une partie concernait les coutumes des Slaves a été écrit dans la première moitié du IX siècle par un certain Jarmi, mais il n'a pas été conservé. Il faut attendre la deuxième moitié du siècle, avec Ibn Khurradadhbeh et al-Mas'udi pour avoir des informations intéressantes et substantielles. 
$\mathrm{KV}$ : De quelle nature étaient ces relations?

JCD : Au début, hormis les contacts belliqueux dus aux premières expéditions musulmanes en Anatolie ou au-delà du Caucase, les relations sont d'abord d'ordre commercial, que ce soit par le commerce des esclaves ou par celui de la fourrure.

Avec la constitution d'États et leur christianisation, ces relations deviennent aussi politiques. Avec la mise en place de relations régulières, commerciales, à partir du début $\mathrm{du} \mathrm{IX}^{\mathrm{e}}$ siècle, on a des relations de voyages ou des impressions de commerçants qui transmettent une expérience vécue de leur contact avec les différentes populations slaves et l'image qu'en avaient les littérateurs arabes s'en affinent d'autant plus.

DW : Les Polonais possèdent un maigre savoir au sujet d'un voyageur exotique Ibrâhîm ibn Ya'qûb qui a décrit le pays des premiers Piast. Connaissons-nous des voyageurs venus d'Orient qui se sont aventurés dans les pays slaves?

JCD : À propos d'Ibrâhîm ibn Ya'qûb, je me permets de préciser que son édition de base reste celle faite par Tadeusz Kowalski en 1946, à Cracovie et qu'en 2008, dans cette même ville, s'est tenu un colloque sur ce sujet, notamment organisé par Andrej Zaborski. Ce texte est d'une importance exceptionnelle d'ailleurs car le voyage du personnage l'a conduit en Europe occidentale et puis en Europe centrale. Le récit d' Ibrâhîm ibn Ya'qûb est remarquable tant dans l'histoire de la littérature géographique arabe que pour les informations qu'il livre. Deux exemples suffiront... Ce voyageur juif, originaire de Tortose, a rencontré à Magdebourg une délégation de Bulgares envoyée auprès de l'empereur Otton $\mathrm{I}^{\mathrm{er}}$. Il a perçu les effets de leur christianisation et de leur slavisation quand il nous dit qu'ils ont traduit l'Évangile en slave. C'est le même auteur qui, en nous décrivant la manière dont les slaves font un bain de vapeur dans leur maison en jetant de l'eau sur un fourneau porté au rouge, utilise le terme d'izba!

Hormis Ibrâhîm ibn Ya'qûb, je citerais en premier lieu Ibn Fadlân qui, au X $\mathrm{X}^{\mathrm{e}}$ siècle, accompagne une ambassade envoyée par Baghdad auprès du khan des Bulgares de la Volga et qui met par écrit sa relation de voyage. Il croise des « Russes » sur la Volga et il est impressionné par leur blondeur, leur teint et leur carrure, mais nous sommes là encore en présence de Scandinaves en voie de slavisation, soit les Varègues. Il y a bien sûr Abû Hâmid al-Gharnâtî qui est resté deux décennies en Europe de l'est dans la deuxième moitié $\mathrm{du} \mathrm{XI}^{\mathrm{e}}$ siècle et qui rencontre des Slaves orientaux, disséminés entre le Dniestr et la Volga. Il note plusieurs traits culturels comme l'utilisation chez eux de fourrures en guise de monnaie ou l'ordalie des sorcières que l'on plongeait dans une rivière. Il les sait chrétiens mais note encore un reliquat de paganisme. Il parvient avec une caravane jusqu'à Kiev, qu'il nomme d'un nom altaïque, « couman » probablement. Notons qu'il y rencontre d'autres musulmans. Pour vous dire la vérité, cet auteur est une mine d'informations pour toutes ces régions car il nous renseigne même sur les populations finno-ougriennes des Manses et des Khanses. Il nous décrit par exemple la chasse à la baleine dans la mer Baltique et nous donne la plus ancienne description d'un ski!

Il faut aussi mentionner Ibn Battûta, un globe-trotter arabe du XIV ${ }^{\mathrm{e}}$, originaire de Tanger et qui se retrouve en Crimée et en Russie méridionale vers 1333, chez le khan de la Horde d'or. Il serait parvenu jusqu'à la ville de Bulgar, sur la Volga - mais il se peut qu'il nous rapporte là un récit collecté auprès de commerçants rencontrés auprès du khan de la Horde d'or. Il nous donne d'ailleurs la description d'un traîneau tiré par des 
chiens et des détails sur le troc pratiqué auprès des populations riveraines de la Volga et de la Kama pour acheter des fourrures.

Plus près de nous, du troisième quart du XVIII ${ }^{e}$ siècle nous avons le récit d'un compagnon du patriarche Makarius III d'Antioche, à savoir Bulus ibn al-Za'im al-Halabî, qui accompagna le patriarche lors d'un long voyage en Moldavie, en Ukraine et en Russie, en quête d'une aide financière pour son église. C'est un récit sobre et factuel qui donne des informations sur les pays inconnus des lecteurs, avec aussi la description des pratiques religieuses des autres chrétiens. L'auteur s'intéresse à l'architecture, aux coutumes, à l'agriculture, aux traditions, à la manière dont le pouvoir fonctionne et s'affiche. Quand il le peut, il fournit des notices historiques, par exemple sur la conversion de Novgorod au christianisme. Comme ce sont des invités de marque, traités comme tels, ils n'ont pas de contact avec le petit peuple. L'auteur finira néanmoins par parler russe.

DW : Que savaient les peuples arabes sur les pays slaves? La perception des Slaves étaitelle mythifiée ? Hantaient-ils l'imaginaire populaire?

JCD : D'entrée de jeu, il faut préciser que la majeure partie de la littérature arabe médiévale conservée émane des milieux savants, ainsi appréhender l'imaginaire populaire est particulièrement difficile voire impossible pour le haut Moyen Âge. Par exemple, un corpus de textes comme celui des Mille et une nuits, qui en l'état peut remonter au XIII siècle mais avec des noyaux plus anciens, ne met jamais en scène de Slaves.

Encore une fois, on retrouve leur mention dans la littérature savante et principalement historico-géographique. N'oublions pas que nous sommes au Moyen Âge et que nos observateurs musulmans voient les populations du monde au travers d'un prisme théorique issu d'un héritage vétérotestamentaire, c'est-à-dire que les nations qui peuplent le monde sont forcément issues de la descendance de Noé et dans le cas des Slaves, ils descendent de Japhet, qui est aussi l'ancêtre des Turcs. Ce qui semble parfaitement logique à nos observateurs puisque ces deux populations sont septentrionales et vivent sous des climats rigoureux analogues. Toujours au niveau des préjugés, nos auteurs ont hérité de la théorie du déterminisme géographique qui finalement, a posteriori, est appelée à la rescousse pour justifier des observations. Ainsi, c'est le climat froid et humide dans lequel vivent les Slaves qui détermine leur complexion, pâteuse. Comme le froid est intense et l'environnement inhospitalier, ils sont farouches et ont des mœurs rudes. Ils ont un teint blanc, la peau fine et les cheveux roux.

Sur une carte politique, les Slaves sont, dès le début, comptés parmi les populations qui peuplent l'Europe, à côté des Francs, des Alains ou encore des Byzantins. Ils occupent un vaste territoire, traversé par plusieurs fleuves qui sont bien identifiés, la Volga, le Don et le Danube. D'une manière générale, nos auteurs perçoivent bien les mouvements de populations qui ont affecté l'Est de l'Europe et globalement, au $\mathrm{X}^{\mathrm{e}}$ siècle, ils présentent les Slaves comme entourés par les Bulgares de la Volga, les Petchenègues, les Magyars, les Bulgares du Danube et les Francs. Quant aux Russes, ce sont leurs expéditions militaires et leur progression vers le sud depuis les rives de la Baltique qui les font connaître aux musulmans. Remarquons que sur certaines cartes arabes, la mer Baltique est connue sous le nom de mer des Varègues. 
Le territoire des Slaves est décrit comme constitué de forêts et de tourbières, ponctué par des villes fortes. Cet environnement leur fournit leur alimentation, faite de lait et de viande, alors que le miel est la base de leur boisson.

KV : Les Slaves constituaient-ils une entité homogène pour l'Orient ? Ou au contraire des peuples distincts?

JCD : Avouons que chez nos auteurs, le terme de "Slave " est parfois utilisé pour qualifier les Magyars ou les Bulgares. En effet, les Slaves proprement dits et les populations qui les avoisinaient constituaient un patchwork d'ethnies que nos auteurs avaient parfois du mal à débrouiller et que les érudits européens, depuis le XIXe siècle, tentent d'identifier. Je ne prendrai que quelques exemples, chez Ibn Rusteh, un encyclopédiste arabe du début $\mathrm{du} \mathrm{X}^{\mathrm{e}}$ siècle, nous avons la description des Slaves, de la population finnoise appelée Mordwa par les Russes et située le long de l'Oka, des Bulgares de la Volga, des Magyars, des Russes antérieurement à leur slavisation, sans oublier les Khazars et les Petchenègues.

À la fin du même siècle, une géographie persane anonyme nous livre entre autres une description des territoires slaves et russes qui a excité l'érudition époustouflante de savants comme Vladimir Minorsky qui y a retrouvé la mention des Slovènes et peutêtre des Croates ainsi que de Sambatas, le nom de Kiev selon Constantin Porphyrogenète, lorsque la ville était encore la capitale des Polianes, comme le rapporte la Chronique de Nestor.

D'une manière générale, en collationnant nos auteurs, nous retrouvons une dispersion des populations slaves depuis les rives de l'Adriatique jusqu'à la mer Baltique, où ils avoisinent avec des populations finnoises, mais les situations sont souvent imprécises. Un encyclopédiste du milieu du $\mathrm{X}^{\mathrm{e}}$ siècle, al-Mas'ûdî, tente d'ailleurs de dresser un tableau clair de ces populations en nous citant, du nord au sud, les Wiltzes, les Stodoranes, les Obodrites, les Dulèbes, les Glomaci, les Serbes blancs, les Moraves, les Croates, les Tchèques, les Guduscani et les Braničewci. Cette énumération nous rappelle au passage que l'histoire ethnique de l'Europe est d'une enivrante complexité que les sources arabes permettent d'éclairer. Al-Mas'udi nous parle aussi de Prague en nous vantant la richesse de son souverain.

Ibrâhîm ibn Ya'qûb, qui écrit dans la deuxième moitié du $\mathrm{X}^{\mathrm{e}}$ siècle, a pour lui d'avoir voyagé en Europe et nous dresse un tableau plus circonstancié en décrivant d'abord le royaume des Obodrites du Mecklembourg dont le souverain est alors Nakon. Il s'arrête ensuite à celui de Boleslav, roi de Bohème, dont la capitale est Prague. Et enfin, il parle du plus vaste des trois royaumes, celui de Mieszko, duc de Pologne. C'est notamment à cette occasion que l'auteur y donne la plus ancienne mention de Cracovie dans une source écrite ! Il termine en mentionnant les Prussiens, alors encore baltes.

Je dois tout de même ajouter que les auteurs arabes d'après le $\mathrm{XI}^{\mathrm{e}}$ siècle, à l'exception d'al-Idrîsî, sont bien moins détaillés ou se limitent à compiler les informations de leurs prédécesseurs.

DW : La revue «Rocznik Orientalistyczny » a publié votre texte sur l'image de la Pologne et I'Europe centrale chez les Orientaux, plus précisément dans le traité Uns al-muhadj wa-rawd al-furadj d'Al-Idrîsî. Comment se présentait cette image?

JCD : Le cas d'al-Idrîsî est tout à fait particulier et sans pareil. Ceci dit en passant, il faut savoir que l'un des manuscrits de l'ouvrage est conservé à Saint-Pétersbourg et un autre à Sofia, en Bulgarie. 
Al-Idrîsî, qui a travaillé en Sicile au XII ${ }^{\mathrm{e}}$ siècle, sous les rois normands Roger II et son fils Guillaume I ${ }^{\mathrm{er}}$, a réalisé deux traités de géographie. Le premier a été écrit à l'instigation de Roger II, d'où le titre qu'on lui donne parfois de «Livre de Roger » et le second, qui est une refonte abrégée du premier avec l'addition d'un nouveau matériel topographique, a sans doute été composé sous Guillaume Ir. Quoi qu'il en soit, la qualité et la quantité des informations que l'auteur a recueillies notamment sur l'Afrique et l'Europe en font une source incomparable pour la géographie urbaine de ces régions, et l'Europe orientale n'y échappe pas. Permettez-moi de rappeler ici la mémoire de Joachim Lelewel qui, vers 1850, publia à Bruxelles une première étude partielle de ce texte à partir d'une traduction française faite par un arabisant français, Amédée Jaubert. Alors que le traducteur avait par endroit très mal compris le texte, Joachim Lelewel - qui ne connaissait pas l'arabe - est parvenu à proposer des identifications de toponymes d'une grande pertinence. Un siècle plus tard en 1945, Tadeusz Lewicki a enfin donné une édition commentée de la partie de ce texte qui concernait la Pologne alors que Stoyanka Kenderova, à Sofia, a consacré une étude détaillée aux cartes des Balkans. Enfin, dernièrement, l'arabisante russe Irina Konovalova s'est penchée sur la description des territoires russes chez al-Idrîsî. A plusieurs reprises, al-Idrîîi y insiste sur le nombre et la qualité des savants chrétiens que renferme la Pologne. Comme il s'intéresse principalement aux localités et à leur prospérité, il mentionne et décrit brièvement Cracovie, Gniezno, Włocławek, la principauté de Sandomierz, Szczecin, Przemyśl. De manière générale, il voit la Pologne comme un beau et vaste pays traversé de nombreux cours d'eau. Il est riche et prospère, avec des villes et des cultures florissantes. À ce propos, l'auteur fait état de l'abondance des vignes et des oliviers, c'est dire que son informateur a sans doute un peu embelli la situation!

Dans son deuxième ouvrage, le Uns al-muhadj, il n'apporte pas d'informations nouvelles sur la Pologne, je me permets cependant de souligner qu'un arabisant finlandais, Oivia Tuulio, a relevé en 1936 qu'al-Idrîsî y donne pour la première fois dans une source arabe le nom « Volga » au cours supérieur du fleuve. Il faut savoir que dans les sources arabes et iraniennes, le cours inférieur du fleuve est connu sous son turc d'Itil.

DW: Un de vos nombreux articles a été consacré au commerce des fourrures entre I'Europe orientale et le Proche-Orient du IX ${ }^{\mathrm{e}}$ au XIII siècle. Nous connaissons aussi I'histoire de la route de l'ambre dont les axes principaux semblent toujours d'actualité. Ma question porte sur vos voyages; avez-vous essayé, en fidèle scientifique, de faire ces voyages à l'image de vos prédécesseurs?

JCD : Pour vous dire la vérité, l'étude de la littérature géographique, quelle qu'elle soit d'ailleurs, est une constante invitation au voyage. L'énumération de villes sur un itinéraire vous pousse à les vérifier sur la carte et finalement à essayer de voir à quoi elles ressemblent. Je pense avoir parcouru la plupart des pays du Maghreb ainsi que du Proche et du Moyen-Orient jusqu'à l'Inde d'ailleurs. Mais, si d'un point de vue émotionnel, c'est une expérience enrichissante, d'un point de vue scientifique, cela n'a guère d'intérêt car la géographie ou la toponymie historiques s'étudient en bibliothèque ! À la limite, le voyage est nécessaire pour aller lire un manuscrit inédit conservé à tel endroit ou pour avoir la copie de tel ouvrage introuvable en Belgique. J'avoue qu'il fut un temps où j'aurais voulu parvenir sur les bords de la mer Noire en traversant toute l'Europe puis l'Ukraine, mais si je réalise un jour ce périple, ce sera en touriste. 
KV : Avez-vous, lors de vos voyages, exploré l'univers slave? Si oui, l'avez-vous observé à travers le prisme de vos lectures orientales?

JCD : J'ai de bons contacts avec mes collègues polonais et je publie d'ailleurs régulièrement dans des revues orientalistes polonaises. Il y a plusieurs années, j'ai participé à un colloque à Cracovie dont j'ai gardé un très bon souvenir, tant pour l'aspect scientifique que pour l'accueil sur place. Entre-temps, je pris des cours de russe pour pouvoir au moins lire les textes de mes collègues. Je vous avoue tout de même que j'ai l'impression que les langues slaves ont une grammaire et une phonétique bien plus compliquées que les langues sémitiques ou romanes !

DW : Avez-vous parfois l'impression de suivre une carte aux trésors?

JCD : Voilà une belle expression! C'est peut-être plus un jeu de piste car, au départ, je n'ai pas tous les éléments en main. À partir d'un texte ou deux, de quelques toponymes, il faut découvrir ou imaginer la démarche intellectuelle de l'auteur qui l'a écrit, puis établir le rapport avec le réel pour l'auteur et ensuite pour nous. Dans ces démarches, on est en droit - et même obligé - d'utiliser toutes les disciplines à notre disposition, de l'archéologie à la philologie. Par exemple, lorsqu'Abû Hâmid nous décrit la construction d'un habitat de castor sur le bord de la Volga, nous sommes amenés à vérifier cette description, de même lorsqu'il nous signale que le jour le plus long à la ville de Bulgar (aujourd'hui Bulgarskoe, près de Kazan') est de 20 heures, il convient d'en calculer la latitude sur cette base, soit un peu plus de $57^{\circ}$ nord. Il nous arrive parfois de rester plusieurs années bloqués sur un court texte car nous sommes incapables, impuissants, à comprendre la réalité qui se cache derrière un mot ou une expression, ou en tout cas pas de manière satisfaisante. On y revient périodiquement en ayant alimenté sa réflexion d'autres lectures et, finalement, une interprétation optimale surgit. Il y a aussi des cas où l'interprétation est boiteuse et on le sait en âme et conscience. Alors, on nuance, on soupèse, on utilise le conditionnel. Autant les cartographes anciens avaient peur de laisser un blanc sur leurs cartes, autant le commentateur moderne craint de devoir avouer son ignorance. La vanité se cache partout...

KV : Sur la route de vos recherches, avez-vous des projets futurs liés à l'étude des contacts entre les Slaves et I'Orient?

JCD : Je viens de terminer une étude qui porte sur la France et les territoires avoisinant dans le deuxième ouvrage de géographie d'al-Idrisi et cela s'est révélé très riche de nouvelles informations. Je caresse dès lors le projet de me remettre à l'étude de la partie du texte qui concerne les terrioires russes au sens large. De même, les cartes d'alIdrisi qui concernent la Russie mériteraient d'être à nouveau étudiées ou, en tout cas, d'être commentées en français ou en anglais car les quelques travaux qui les concernent ont été rédigés essentiellement en russe, ce qui en limite la diffusion.

Cependant, ne me demandez pas quand je compte mener à terme ces entreprises car j'ai parfois l'impression de me lancer dans une nouvelle recherche à chaque fois que je sors de mon lit et comme nos heures sont finalement comptées, des chantiers resteront inachevés...

DW et KV: Nous vous remercions vivement au nom de la rédaction de «Slavica Bruxellensia » et de nos lecteurs d'assouvir si élégamment notre curiosité sur des thèmes 
passionnants et toujours trop peu connus. Et nous vous souhaitons encore de belles et nombreuses découvertes dans le domaine de vos recherches.

\section{BIBLIOGRAPHIE}

Ducène J.-Ch., Ducène J.-Ch., De Grenade à Bagdad, la relation de voyage d'Abû Hâmid al-Gharnâtî (1080-1168), traduction annotée, L'Harmattan, (Histoire et perspectives méditerranéennes), Paris, 2006, 210 p.

Ducène J.-Ch., L'Afrique dans le Uns al-muhağ wa-rawd al-furağ d'al-Idrīsī, Édition, traduction et commentaire, Peeters, Leuven, 2010, lvi et 249 p.

\section{INDEX}

Index géographique : Russie

Mots-clés : cartographie arabe, cartographie slave, géographie arabe, géographie slave

\section{AUTEURS}

\section{DOROTA WALCZAK}

Responsable de la Chaire de Polonais, section de Langues et Littératures modernes, option Slaves, de l'Université Libre de Bruxelles (Belgique) ; Rédactrice en chef de « Slavica Bruxellensia »

\section{KATIA VANDENBORRE}

Doctorante à l'Université Libre de Bruxelles (Belgique) et à l'Université de Varsovie (Pologne) ; membre du comité de rédaction de « Slavica Bruxellensia » 